LAWRENCE LIVERMORE N A T IO N A L LABORATORY

\title{
Accessing High Pressure States Relevant to Core Conditions in the Giant Planets
}

B. A. Remington, R. M. Cavallo, M. J. Edwards, D. D. Ho, K. T. Lorenz, H. E. Lorenzana, B. F. Lasinski, J. M. McNaney, S. M. Pollaine, R. F. Smith

May 10, 2004

5th International Conference on High Energy Density Laboratory Astrophysics

Tucson, AZ, United States

March 10, 2004 through March 13, 2004 
This document was prepared as an account of work sponsored by an agency of the United States Government. Neither the United States Government nor the University of California nor any of their employees, makes any warranty, express or implied, or assumes any legal liability or responsibility for the accuracy, completeness, or usefulness of any information, apparatus, product, or process disclosed, or represents that its use would not infringe privately owned rights. Reference herein to any specific commercial product, process, or service by trade name, trademark, manufacturer, or otherwise, does not necessarily constitute or imply its endorsement, recommendation, or favoring by the United States Government or the University of California. The views and opinions of authors expressed herein do not necessarily state or reflect those of the United States Government or the University of California, and shall not be used for advertising or product endorsement purposes.

This work was performed under the auspices of the U.S. Department of Energy by the University of California, Lawrence Livermore National Laboratory under Contract No. W-7405-Eng-48. 


\title{
Accessing high pressure states relevant to core conditions in the giant planets
}

\author{
B.A. Remington, R.M. Cavallo, M.J. Edwards, D.D.-M. Ho, K.T. Lorenz, \\ H.E. Lorenzana, B.F. Lasinski, J.M. McNaney, S.M. Pollaine, R.F. Smith
}

In preparation for submittal to Astrophysics \& Space Science, for the proceedings of the 5th HEDLA-2004 conference

April 14, 2004, 8:20 am

\begin{abstract}
:
We have designed an experimental technique to use on the National Ignition Facility (NIF) laser to achieve very high pressure $\left(\mathrm{P}_{\max }>\right.$ $10 \mathrm{Mbar}=1000 \mathrm{GPa})$, dense states of matter at moderate temperatures $(\mathrm{kT}$ $<0.5 \mathrm{eV}=6000 \mathrm{~K})$, relevant to the core conditions of the giant planets. A discussion of the conditions in the interiors of the giant planets is given, and an experimental design that can approach those conditions is described.
\end{abstract}

The interior structures of the giant planets, Jupiter, Saturn, Uranus, and Neptune, [Guillot, 1999] and the newly discovered extrasolar planets [Lissauer, 2002] is determined by the compressibility of their constituent matter under the inwardly directed pressure due to gravitation. In laboratory terms, this compressibility corresponds to the high pressure equation of state (EOS) of matter along an isentrope. The EOS and properties of matter at the extreme pressures and densities found in the interiors of the giant planets, however, are quite uncertain. The pressures of interest along an isentrope 
range from 1-8 Mbar in Uranus and Neptune, 1-10 Mbar or more in Saturn, with pressures at the core conditions of Jupiter exceeding 40 Mbar, as shown in Fig. 1. [Guillot, 1999] Under these conditions, dense plasmas are both strongly coupled and Fermi degenerate. [Van Horn, 1991]

Strongly coupled plasmas are typically characterized by the dimensionless parameter, $\Gamma=(Z e)^{2} / a k T$, where $a$ is a characteristic separation distance between ions, $Z e$ is the ion charge state, and $k T$ is the temperature in units of energy. In plasmas with $\Gamma<<$ 1, thermal effects dominate and the plasma is considered "ideal." When $\Gamma \approx 1$, the Coulomb interactions become an equal player, and the plasma enters the strongly coupled regime. When $\Gamma>178$, the plasma becomes so strongly coupled that the ions freeze solid into a crystal lattice. [Van Horn, 1991] Also, when the densities are high enough or temperatures low enough that $k T<\varepsilon_{\mathrm{F}}$, where $\varepsilon_{\mathrm{F}}=p_{F}{ }^{2} / 2 m_{\mathrm{e}}=(1 / 8)(3 / \pi)^{2 / 3}\left(h^{2} / m_{\mathrm{e}}\right) n_{\mathrm{i}}{ }^{2 / 3} \propto \rho^{2 / 3}$ is the Fermi energy $\left(p_{\mathrm{F}}\right.$ is the Fermi momentum set by the Pauli exclusion priciple and $n_{\mathrm{i}}$ is the ion number density), the plasma is called degenerate. Here, electron degeneracy pressure becomes a major part of the total pressure. The isentropes for the giant planets indicate that the plasma in their interiors is both strongly coupled and highly degenerate. Hence, the internal structure, $\rho(r), T(r)$, of the giant planets is determined by the EOS of dense, degenerate plasma mixtures at very high pressures, $\mathrm{P}=1$ to $40 \mathrm{Mbar}$, and moderate temperatures, $\mathrm{kT}<\sim 1 \mathrm{eV}$.

Different theories of the EOS of dense, high pressure matter lead to different predictions about the interior conditions of the giant planets, [Saumon, 2004] and more generally, differentiate between different planetary formation models [Guillot, 1997]. The EOS of dense, strongly coupled, degenerate plasma is notoriously difficult to calculate from first-principles theories, however, and many uncertainties remain. One of the fundamental open questions about the interior of Jupiter, for example, is whether there is a first order plasma phase transition (PPT) between a molecular, dielectric 
hydrogen mantle and a monatomic, ionized (conducting) hydrogen core at a radius of $\sim 0.75$ jovian radii $\left(R_{\mathrm{J}}\right)$. Another fundamental open question is whether the He and other higher-Z elements in solution with $\mathrm{H}$ condense out (become immiscible) into droplets at sufficiently high pressures and densities. These condensation droplets would sink radially inwards like rain, releasing gravitational potential energy, and serve as an internal heat source for the planet. These two questions relate primarily to Jupiter and Saturn, whose constituents are dominated by $\mathrm{H}$ and He. In the case of the "ice giants" Uranus and Neptune, the uncertainties are even larger. These planets contain much larger fractions of heavier elements, such as $\mathrm{CH}_{4}, \mathrm{NH}_{3}$, and $\mathrm{H}_{2} \mathrm{O}$, largely in the form of ice, whose properties and EOS at very high pressures and densities are known even less.

Experiments are vital to improve our understanding of the interior structures of the giant planets. The most widely used experimental technique for determining highpressure EOS is through the use of shock waves to determine the principle Hugoniot of materials. [Collins, 1998; Knudson, 2003; Boehly, 2004] The difficulty with this technique as concerns planetary interiors, however, is that it produces compressed states of matter at too high a temperature. What is needed is an experimental technique to produce high pressure compression at lower temperatures, closer to an isentrope. We report here on our designs to use the National Ignition Facility (NIF) laser [Hogan 2001] to create a very high pressure, nearly isentropic "drive" to access conditions approaching those at the cores of the giant planets.

We illustrate in Figure 2a the experimental configuration we are designing for achieving dense high-pressure $(>10 \mathrm{Mbar})$ states at moderate temperatures $(\mathrm{kT}<\sim 0.5$ $\mathrm{eV}$ ), using the NIF laser [Hogan, 2001] as the energy source. The target in this case was assumed to be a $1.6 \mathrm{~mm}$ thick carbon "reservoir" at density of $1.0 \mathrm{~g} / \mathrm{cm}^{3}$ followed by a 2 mm vacuum gap, then the "payload". The payload consists of a thin $\mathrm{CH}(2 \% \mathrm{Br})$ heat shield at density of $1.2 \mathrm{~g} / \mathrm{cm}^{3}$, followed by the sample under study, then a tamper 
material. For the purposes of roughing out a design to establish what pressures, compressions, and temperatures were possible as a function of time, and to determine the approximate laser and experimental conditions required, the sample was assumed to be $100 \mu \mathrm{m}$ thick Ta, backed by a thick ( $500 \mu \mathrm{m})$ Mo tamper. The laser was assumed to be incident on the front face of the reservoir (ie, the side facing away from the sample) at an intensity of $6.5 \times 10^{13} \mathrm{~W} / \mathrm{cm}^{2}$ in a $78 \mathrm{~ns}$ square pulse in a $3.5 \mathrm{~mm}$ diameter flat spot. This would require $0.6-0.9 \mathrm{MJ}$ of laser energy using half of NIF in single-sided illumination to drive the compression wave, which just fits within the predicted performance specifications of NIF. This leaves the other half of NIF to drive backlighters, that is, separate synchronized sources of hard x-rays for diagnosis.

The ensuing dynamics are illustrated schematically in Fig. 2b. The laser pulse ablatively drives a strong shock through the low-Z reservoir (see curve ' $t_{1}$ ' in Fig. 2b). When the shock reaches the back side (the side opposite from where the laser was incident), the reservoir unloads into vacuum as plasma "ejecta" (see curve ' $t_{2}$ ' in Fig. 2b). The pressure that is applied to the sample results from the increasing ram pressure, $P_{\mathrm{ram}}=$ $\rho_{\text {ejecta }} \mathrm{V}^{2}{ }_{\text {ejecta }}$, as the ejecta plasma stagnates and accumulates on the payload (see curve ' $\mathrm{t}_{3}$ ' in Fig. 2b). This pressure increases smoothly and monotonically in time as the reservoir unloads, until the reservoir material is depleted. We have successfully demonstrated this laser-based technique for generating a high pressure ramped load at the Omega laser over a range of pressures spanning $P_{\max }=0.1-2$ Mbar. [Edwards, 2004; Lorenz, 2004] Initial measurements of the EOS of $\mathrm{Al}$ with such a laser-driven ramp wave appear to compare very favorably with results from more conventional techniques. [R. Smith, 2004] This laser-based technique for generating a ramped pressure wave was motivated by the early 
work of Barnes using high-explosive (HE) as the energy source for driving the shock through the reservoir.[Barnes, 1974] More recently, a technique using the high current pulse at the $\mathrm{Z}$ magnetic pinch facility at SNLA has also demonstrated the ability to generate high pressures ( 1 Mbar) with a shockless, quasi-isentropic drive. [Reisman, 2001; Hall, 2001; Cauble, 2002]

In Fig. 3 we show the results from simulations of the design illustrated in Fig. 2. The pressure versus time at the $\mathrm{CH}(\mathrm{Br})-\mathrm{Ta}$ interface is given in Fig. 3a, showing that a peak pressure of $\sim 10 \mathrm{Mbar}\left(10^{3} \mathrm{GPa}\right)$ is reached, with a $25-30 \mathrm{~ns}$ rise time. To achieve this "gentle" rise time, we assumed in this design $0.5 \mathrm{eV}$ of preheat in the reservoir, so that the back side started to release into the gap, creating a density ramp prior to the arrival of the main reservoir shock. Subsequent designs have replaced the preheat with a graded density profile in the reservoir on the side facing the gap, with equivalent results. The pressure versus time curve shown in Fig. 3a shows no evidence of any strong shocks, but rather exhibits a smoothly increasing applied pressure with time. The pressure and temperature conditions at peak compression, $\mathrm{P} \sim 10$ Mbar and $\mathrm{T} \sim 0.3-0.5 \mathrm{eV}$, are similar to those in the cores of Saturn, Uranus, and Neptune, but fall short of the pressures at the core of Jupiter.

The final point to consider is whether this experimental design for NIF is approaching sufficiently the isentropic conditions of planetary interiors. We examine this issue in two ways. We first plot in Fig. 3b the pressure at a position $25 \mu \mathrm{m}$ into the Ta (from the drive side) as a function of compression, $\rho / \rho_{0}$, both during the compression phase and also throughout the release phase of the drive, as compared to the cold curve (ie, the true isentrope). The initial state of the Ta in both cases was $\rho_{0}=16.7 \mathrm{~g} / \mathrm{cm}^{3}$ and 
$\mathrm{T}_{0}=10^{-3} \mathrm{eV}$. The deviation from the isentrope upon release (after loading to $10 \mathrm{Mbar}$ ) of the simulated pressure is small. For these dense, degenerate conditions, however, pressure is mainly determined by density, and pressure is not a good indicator of the heating which causes departures from the isentrope.

In Fig. 3c we plot temperature, kT (eV) versus compression, $\rho / \rho_{0}$, at locations of $10 \mu \mathrm{m}$ (top two curves, A and E), $25 \mu \mathrm{m}$ (curves, B and F), $50 \mu \mathrm{m}$ (curves $\mathrm{C}$ and G), and 75 (curve $\mathrm{D}$ and $\mathrm{H}$ ) $\mu \mathrm{m}$ into the Ta sample, again compared to the isentrope (bottom curve). This plot shows much more sensitivity to the heating effects. The dashed curves correspond to simulations that include material strength via the Steinberg-Guinan constitutive model. [Steinberg, 1980] The solid curves correspond to simulations where strength has been turned off. First consider the dashed curves, representing the simulations including material strength. Starting with the dashed curves corresponding to locations deepest into the sample (curves $\mathrm{H}$ and $\mathrm{G}$ ), the $\delta(\mathrm{kT}) \sim 0.07-0.08 \mathrm{eV}$ of heating above the isentrope evident by compression of $\rho / \rho_{0} \approx 1.4$ is likely caused by the ramped pressure pulse steepening into a slight shock at this depth into the sample. The temperature of $\mathrm{kT} \sim 0.3 \mathrm{eV}$ at peak compression for curves $\mathrm{D}$ and $\mathrm{H}$ are about a factor of $\sim 5$ below the predicted melt temperature, based on a Lindemann law estimate. [Steinberg, 1980] The curves $\mathrm{E}$ and $\mathrm{F}$ in Fig. 3c correspond to locations in the Ta that are closer to the $\mathrm{CH}(\mathrm{Br})$ heat shield. These curves display a distinctly different behavior. Their trajectories on compression up to $\rho / \rho_{0} \sim 2$ follow rather closely curves $\mathrm{G}$ and $\mathrm{H}$ representing locations deeper in the sample. During the final compression to 2.2 and throughout their release, however, these two curves show a distinct increase in temperature. This is due to a conductive heat wave advancing from the hot stagnating 
plasma impacting the $\mathrm{CH}(\mathrm{Br})$ heat shield. Subsequent designs should utilize thicker heat shields, to reduce this source of heating. We note that at $\rho / \rho_{0}=2.2$, the Lindeman law implies a melt temperature for Ta of $1.4 \mathrm{eV}$, which is considerably higher than any of the curves shown in Fig. 3c. In this design, the Ta sample is predicted to remain in the solid state while under compression, meaning that it retains material strength. This strength will become a source of heating, as work is done against the strength of the sample in driving the deformation (compression). By comparing the solid versus dashed curves in Fig. 3c, which correspond to identical simulations with strength turned on or off, we see that heating due to strength is predicted to be a $10-15 \%$ effect. Solid-state properties, such as phase, elastic constants, shear modulus, and strength under these extreme conditions of pressure, temperature, compression, and strain rate would be of significant scientific interest. [Soderlind, 1998]

In conclusion, we are developing an experimental capability for creating ramped pulse compressions reaching peak pressures over the range of 1-30 Mbar at moderate temperatures $(\mathrm{kT}<\sim 1 \mathrm{eV})$. This suggests that conditions relevant to the interiors of Saturn, Uranus, and Neptune all the way to their cores and much of Jupiter could be experimentally accessed. A NIF design reaching 10 Mbar peak pressure has been shown. More recent NIF designs show that such a ramped drive can achieve peak pressures well over 20 Mbar, and possibly even greater than 30 Mbar, which is approaching the core conditions of Jupiter. We hope in the future that such very high pressure, quasiisentropic drives can be developed and utilized for investigating the properties of matter under conditions relevant to the high pressure interiors of the giant planets and extrasolar planets. 


\section{References:}

1., J. Edwards, K.T. Lorenz, B.A. Remington, S. Pollaine, J. Colvin, D. Braun, B.F.

Lasinski, D. Reisman, J. McNaney, J.A. Greenough, R. Wallace, H. Louis, and D.

Kalantar, Phys. Rev. Lett. 92, 075002 (2004).

2., B.A. Remington, G. Bazan, J. Belak, E. Bringa, M. Caturla, J. Colvin, M.J. Edwards, S.G. Glendinning, B. Kad, D.H. Kalantar, M. Kumar, B.F. Lasinski, K.T. Lorenz, J.

McNaney, M.A. Meyers, S.M. Polaine, D. Rowley, M.S. Schneider, J. Stolken, J. Wark,

S.V. Weber, W.G. Wolfer, and B. Yaakobi, Metallurgical and Materials Transactions A, in press (2004).

3., Tristan Guillot, Science 286, 72 (1999).

3.5, Guillot et al., Icarus 130, 534 (1997).

4., D. Saumon and T. Guillot, LANL interior report LA-UR-040006, submitted to Ap. J. (2004)

5., K.T. Lorenz et al., J. Appl. Phys., to be submitted (2004).

6., H.M. Van Horn, Science 252, 384 (1991)

7., J. Barns, J. Appl. Phys. 45, 727 (1974).

8., J.J. Lissauer, Nature 419, 355 (Sept. 26, 2002).

9a., D.B. Reisman, A. Toor, R.C. Cauble, et al., J. Appl. Phys. 89, 1625-1633 (Feb. 1, 2001).

9b., C.A. Hall, J.R. Asay, M.D. Knudson et al., Rev. Sci. Instrum. 72, 3587 (Sept. 2001).

9c., R. Cauble, D.B. Reisman, J.R. Asay, et al., J. Phys.-Condens. Mat. 14, 10821 (Nov. 11, 2002). 
10., W.J. Hjogan, E.I. Moses, B.E. Warner, M.S. Sorem, and J.M. Soures, Nucl. Fusion 41, 567 (2001).

11., D.J. Steinberg, S.G. Cochran, and M.W. Guinan, J. Appl. Phys., 51, 1496 (1980).

12., P. Soderlind and J. Moriarty, Phys. Rev. B 도, 10340 (1998).

13., Ray Smith, private communication (2004).

14., G. Collins et al., Science 281, 1178 (1998).

15., M. Knudson et al., Phys. Rev. Lett. 90, 035505 (2003).

\section{Figure Captions:}

Fig. 1. Schematic representation of the interiors of Jupiter, Saturn, Uranus, and Neptune. The hashed region indicates a possible radiative zone [in Jupiter, it corresponds to $P$ 0.15 to $0.6 \mathrm{GPa}, T \sim 1450$ to $1900 \mathrm{~K}$, and $R \sim 0.990$ to $0.984 R_{\mathrm{J}}$; in Saturn, it is located around $P \sim 0.5 \mathrm{GPa}, T \sim 1700 \mathrm{~K}$, and $R \sim 0.965 R_{\mathrm{S}}$, but it is probably very marginal]. The range of temperatures for Jupiter and Saturn is for models neglecting the presence of the inhomogeneous region. Helium mass mixing ratios $Y$ are indicated. In the case of Saturn, it is assumed that $Y /(X+Y)=0.16$ in the molecular region. The size of the central rock and ice cores of Jupiter and Saturn is very uncertain. Two representative models of Uranus and Neptune are shown, but their actual interior structure may be significantly different. The figure is reproduced from [Tristan Guillot, Science 286, 72 (1999)].

Fig. 2 (a) Laser-target configuration for the laser-driven ramped-wave compression. The target consists of a $1.6 \mathrm{~mm}$ thick, $1 \mathrm{~g} / \mathrm{ccm}^{3} \mathrm{C}$ reservoir, a $2 \mathrm{~mm}$ gap, and a $100 \mu \mathrm{m}$ thick Ta sample, behind a thin "heat shield" of $\mathrm{CH}(2 \% \mathrm{Br})$, and tamped on its back side with thick Mo. (b) Schematic showing the dynamics of this drive.

Fig. 3. Theoretical design for a shockless, ramped pressure pulse ("drive") for the full NIF laser, utilizing about 0.5 MJ of laser energy. (a) Pressure in the leading zones of the 
Ta vs time (red curve B), showing a peak pressure of $\sim 10$ Mbar at a rise time of 25-30 ns. The black curve (curve A) shows the isentrope. (b) Pressure versus compression at a location of $25 \mu \mathrm{m}$ into the Ta (from the drive side). (c) Temperature versus compression for locations of $10 \mu \mathrm{m}$ (curves A and E), $25 \mu \mathrm{m}$ (curves B and F), $50 \mu \mathrm{m}$ (curves $\mathrm{C}$ and $\mathrm{G}$ ), and $75 \mu \mathrm{m}$ (curves $\mathrm{D}$ and $\mathrm{H}$ ) into the Ta sample. The bottom black curve is again the isentrope. The solid curves assume no material strength, whereas the dashed curves correspond to simulations including material strength through the Steinberg-Guinan constitutive model. 


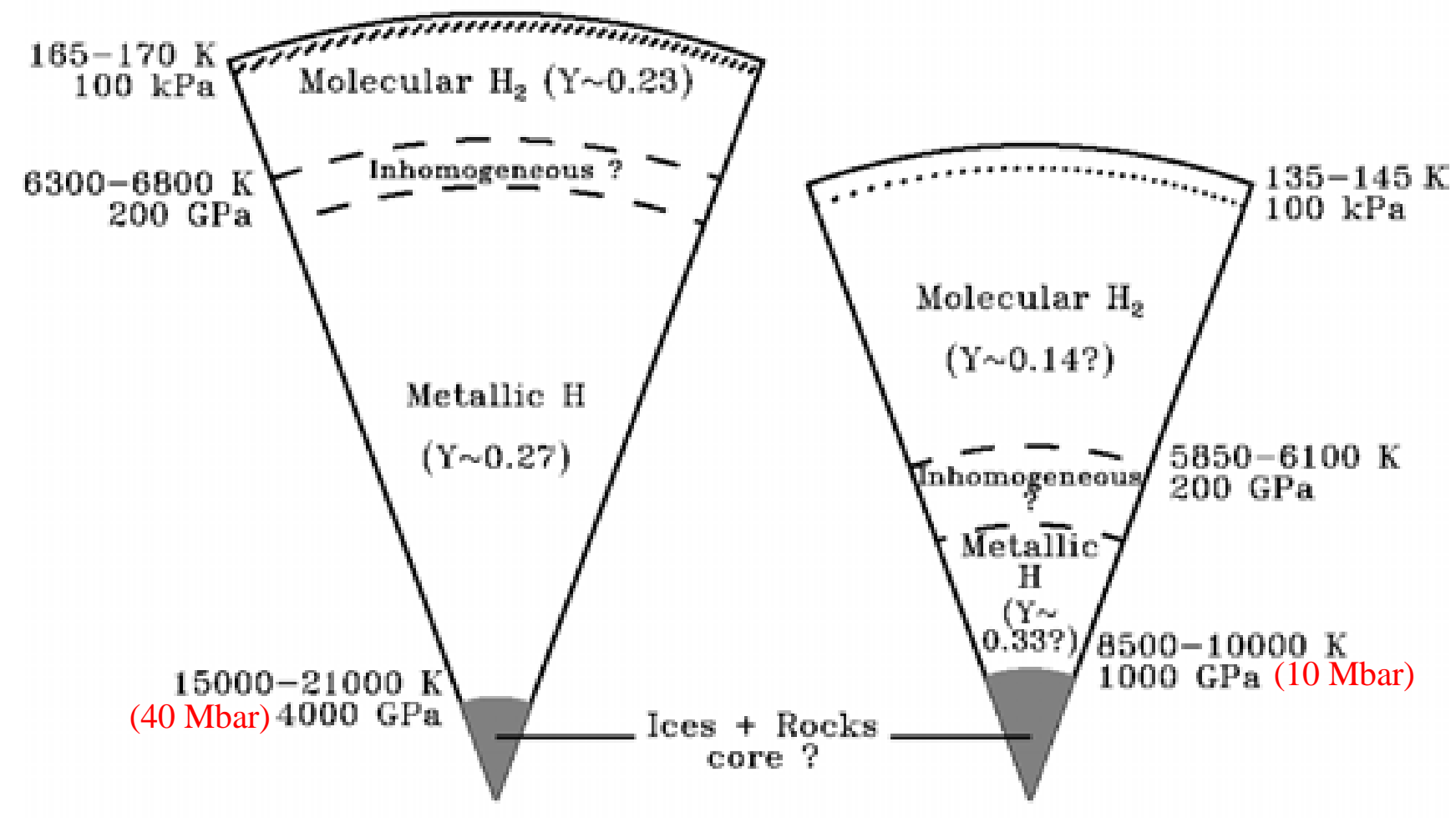

Jupiter

Saturn

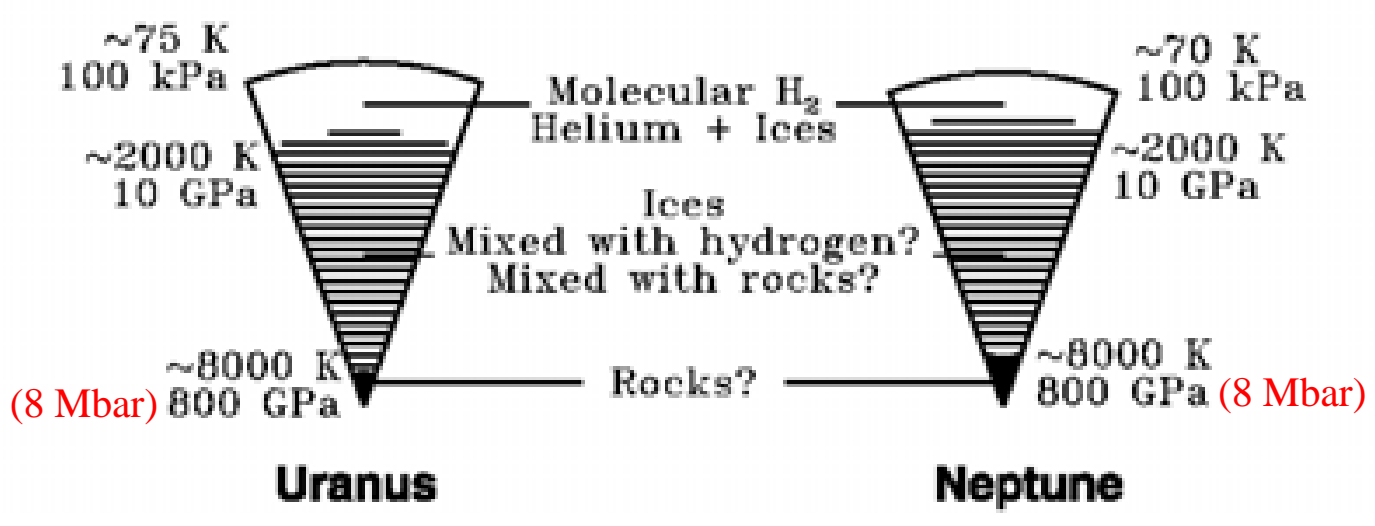

Figure 1

\section{Uranus}


(a)

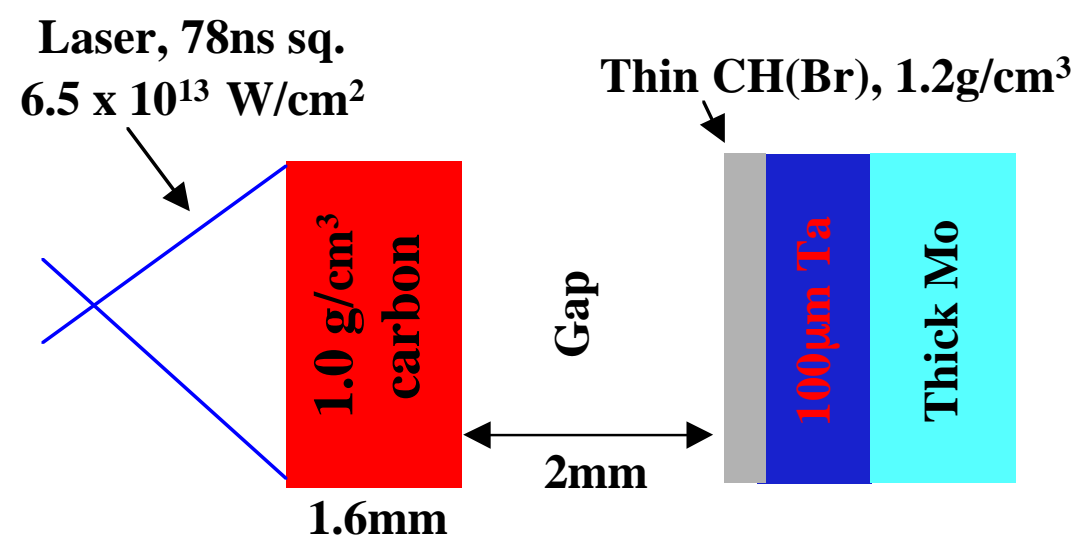

b)

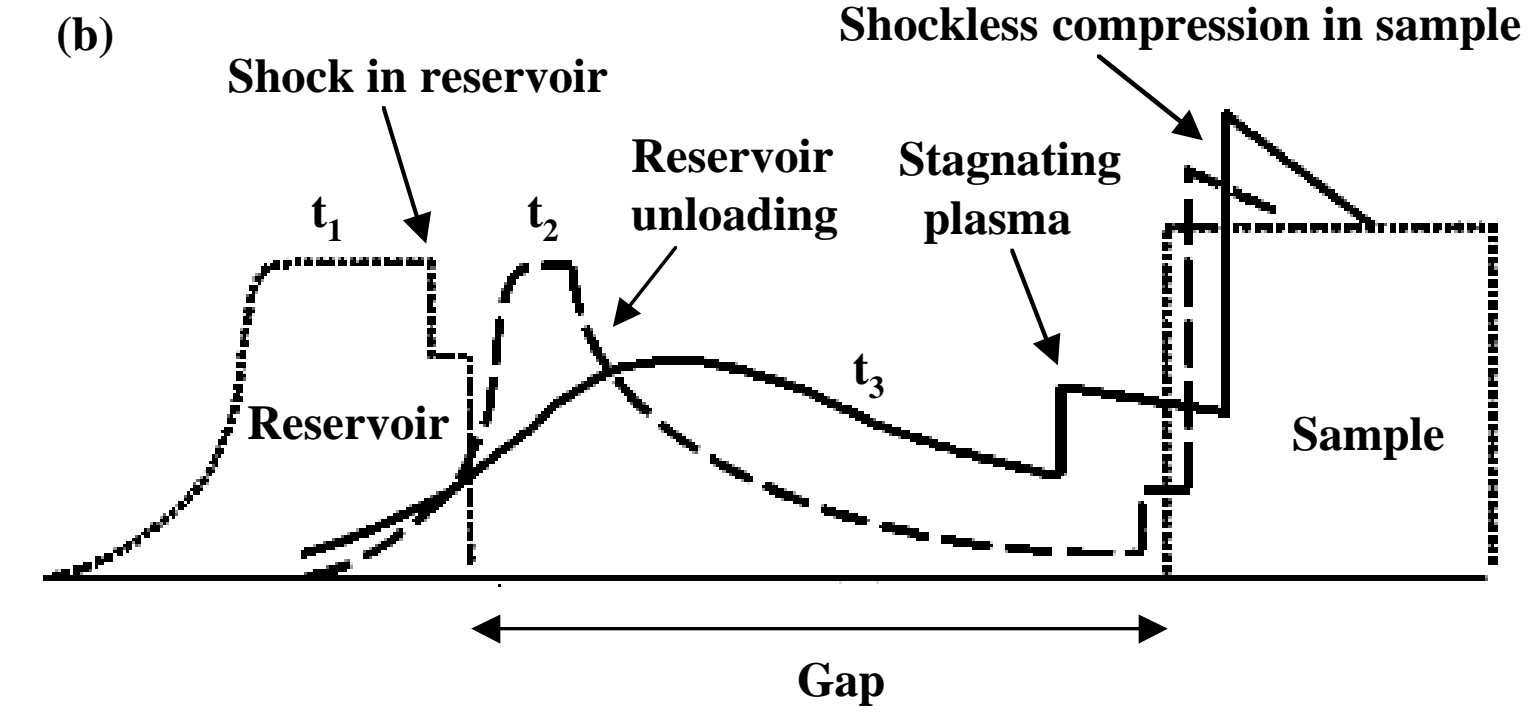

Figure 2 

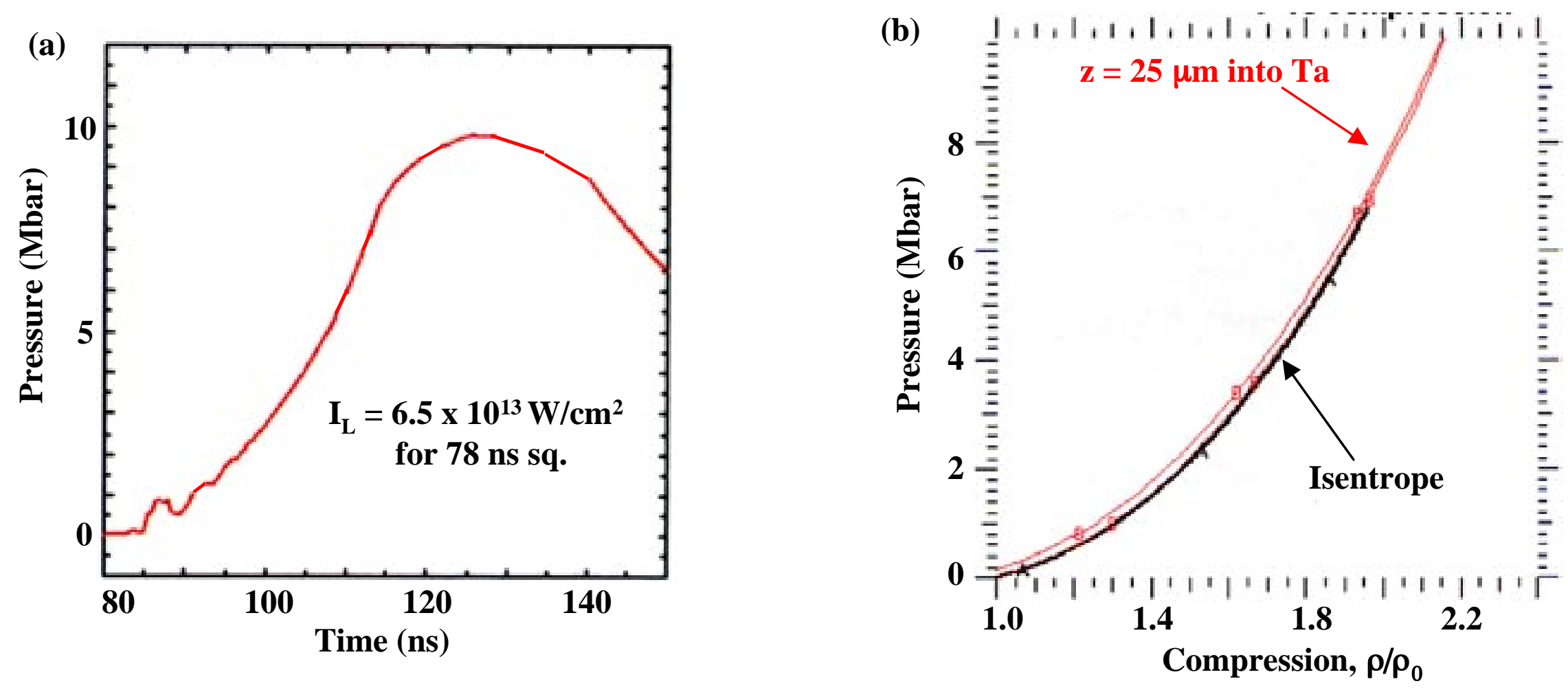

Figure 3 


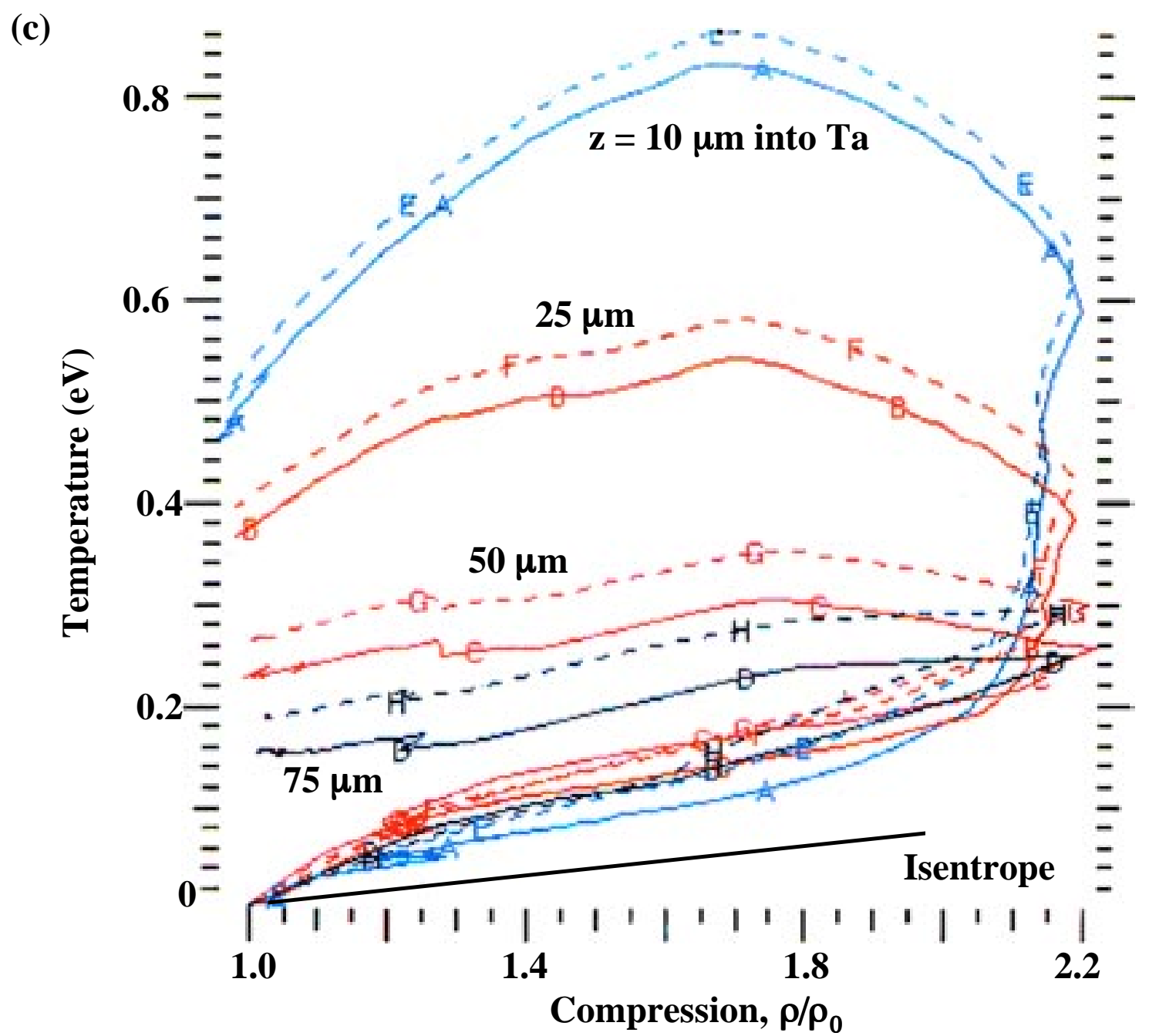

Figure 3 (continued) 